\title{
Facilitated diffusion on mobile DNA: configurational traps and sequence heterogeneity
}

\author{
C. A. Brackley, M. E. Cates, D. Marenduzzo \\ SUPA, School of Physics and Astronomy, University of Edinburgh, Mayfield Road, Edinburgh, EH9 3JZ, UK
}

\begin{abstract}
We present Brownian dynamics simulations of the facilitated diffusion of a protein, modelled as a sphere with a binding site on its surface, along DNA, modelled as a semi-flexible polymer. We consider both the effect of DNA organisation in 3D, and of sequence heterogeneity. We find that in a network of DNA loops, as are thought to be present in bacterial DNA, the search process is very sensitive to the spatial location of the target within such loops. Therefore, specific genes might be repressed or promoted by changing the local topology of the genome. On the other hand, sequence heterogeneity creates traps which normally slow down facilitated diffusion. When suitably positioned, though, these traps can, surprisingly, render the search process much more efficient.
\end{abstract}

PACS numbers:

In living cells, proteins routinely need to reach a target positioned on the DNA, e.g. to initiate transcription of one gene, or to silence or suppress another. Importantly, the search for the target has to be both rapid and efficient. Most experimental results suggest that, within bacterial cells, this process takes about two orders of magnitude less time than one would estimate by assuming unbiased 3D protein diffusion [1-3]. How is such an efficient search realised in practice? The commonly accepted theory is that when seeking their target, proteins alternate between phases of free diffusion through the cytoplasm, and phases in which they slide along the DNA, effectively performing 1D diffusion along its backbone [4 6$]$. This combined strategy is known as "facilitated diffusion" $7-12$.

A simple scaling argument [8] to predict the magnitude of the mean search time, $\tau_{s}$, that a protein needs to find a target on the DNA, is as follows. The key parameters are the DNA length $L$, the volume of the cell $V$, the $3 \mathrm{D}$ and $1 \mathrm{D}$ diffusion coefficients, respectively $D_{3}$ and $D_{1}$ (experiments suggest $D_{1}<D_{3}[13]$ ), and, crucially, the "sliding length", $l_{s}$. This is defined as the typical length of DNA which the protein explores during one episode of 1D diffusion. Via dimensional analysis, one can estimate a typical time spent on a $3 \mathrm{D}$ excursion as $\tau_{3 \mathrm{D}} \sim V / D_{3} L$, while a typical sliding time is $\tau_{1 \mathrm{D}} \sim l_{s}^{2} / D_{1}$. Furthermore, the mean number of 1D-3D search rounds is $N_{s} \sim L / l_{s}$ 14]. One can combine these formulae to estimate $\tau_{s}$ by summing the time spent performing $3 \mathrm{D}$ and $1 \mathrm{D}$ diffusion,

$$
\tau_{s}=N_{s}\left(\tau_{1 \mathrm{D}}+\tau_{3 \mathrm{D}}\right) \sim A \frac{V}{D_{3} l_{s}}+B \frac{L l_{s}}{D_{1}},
$$

where $A$ and $B$ are geometry-dependent constants which cannot be inferred from simple scaling [8, 15]. The most important result from the theory is that there is an optimal sliding length which minimises $\tau_{s}$, given by $l_{s}^{*}=\sqrt{\left(A D_{1} V\right) /\left(B D_{3} L\right)}$. With typical parameters for bacteria and assuming $A \simeq B$ one finds that $l_{s}$ is a few tens of $\mathrm{nm}$.

While appealing, theoretical approaches building on Eq. (1) commonly rely on several approximations in order to make progress. Analytical models usually schematise DNA as a structureless polymer (or assume that the polymer configuration changes on a timescale much quicker than that of the protein movement [12]), and also neglect intersegmental transfers, whereby the protein moves directly (i.e. without a $3 \mathrm{D}$ excursion) between two DNA regions which are close in 3D space, but can be far apart along the DNA backbone. On the other hand, simulations 15 19], usually treat the DNA as frozen (an exception is the lattice study in [18]), and disregard the base pair sequence of DNA.

Here we present a coarse grained simulation of the search process where we relax these two drastic approximations: we include the dynamics of all components (DNA and proteins), and we consider a heterogeneous DNA. We find that both aspects are crucial players in determining how fast facilitated diffusion is. First, we analyse the search process on a string of rosettes, which better represents the conformation of prokaryotic DNA as inferred from experiments [20, 21]. We find that the relative position of the target with respect to the network may change $\tau_{s}$ by orders of magnitude. This giant effect cannot be captured by the theory in Eq. (1), in which the target placement is immaterial. These findings suggest that by changing the local DNA conformation it should be possible to silence or express a given gene. Second, if the DNA-protein interaction is sequence-dependent [22], in general this slows down facilitated diffusion. However, through a careful design of the DNA sequence, we show that one can create a diffusional "funnel" that drives the protein to its target much more quickly.

In this work we used Brownian dynamics (BD) simulations in which we coarse grained DNA as a bead-andspring polymer. Each of the $N$ beads in the DNA had a diameter $\sigma \sim 2.5 \mathrm{~nm}$, and neighbouring beads were connected by finitely extensible nonlinear elastic (FENE) springs. Proteins were modelled as spherical particles with a diameter of $3 \sigma$, with a spherical patch of radius $\sigma$ centred $1.1 \sigma$ away from the protein centre (Fig. 1A). Only the latter was sticky for the DNA, via a (truncated) Lennard-Jones (LJ) interaction. All other coarse-grained 

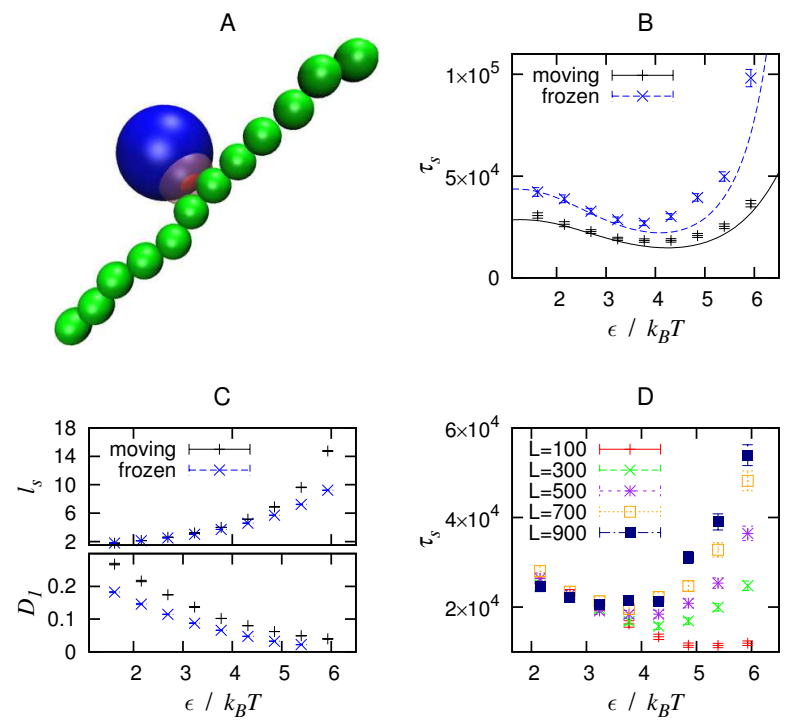

FIG. 1: (A) Snapshot of a DNA segment and a model protein. (B) Mean search time $\tau_{s}$ for frozen and mobile DNA, as a function of DNA-protein affinity, $\epsilon$. Parameters are $L=500 \sigma, V \simeq 50000 \sigma^{3}$ (the fraction of the volume occupied by the DNA is therefore $\simeq 1 \%$ ), while data were averaged over 500 search runs. The lines are a fit of the data with Eq. (11) 26]. (C) Plots of the 1D diffusion coefficient, $D_{1}$, and the sliding length, as a function of $\epsilon$. (D) Dependence of $\tau_{s}$ on affinity for various DNA lengths, $L$ (in units of $\sigma$ ), with fixed $V \simeq 50000 \sigma^{3}$.

beads interact via a purely repulsive potential which captures steric effects (this is achieved by truncating a LJ potential at a mutual distance of $2^{1 / 6} \sigma$ ). Finally, three neighbouring beads along the DNA are subjected to an additional force which models DNA semi-flexibility. Such a force comes from the gradient of the Kratky-Porod potential 23]; this can be expressed as $K \cos \theta$, where $K=k_{B} T l_{p} / \sigma\left(l_{p}=20 \sigma\right.$ for DNA $)$, and $\theta$ is the angle between the three neighbouring beads.

We will refer to the full potential, including both LJ, Kratky-Porod and FENE terms, as $U$. If we denote the position of the $i$-th sphere in the simulation as $\mathbf{x}_{i}$, its evolution is determined by the following Langevin equation,

$$
m_{i} \frac{d^{2} \mathbf{x}_{i}}{d t^{2}}=-\gamma_{i} \frac{d \mathbf{x}_{i}}{d t}-\nabla_{i} U+\sqrt{2 k_{B} T \gamma_{i}} \boldsymbol{\xi}_{i}(t)
$$

where $\gamma_{i}$ is the friction felt by the particle, $\nabla_{i}=\frac{\partial}{\partial \mathbf{x}_{i}}, k_{B}$ is the Boltzmann constant, $T$ is the temperature, $m_{i}$ is the mass of the $i$-th bead, and $\boldsymbol{\xi}_{i}(t)$ is an uncorrelated Gaussian noise with zero mean and unit variance 24]. All simulations were performed via the LAMMPS code 25].

Fig. 1B shows the mean search time as a function of the DNA-protein affinity $\epsilon$ (the depth of the attractive LJ potential, measured in units of $k_{B} T$ ), for the cases in which the DNA is either frozen (into a randomly chosen equilibrium configuration) or mobile. Since the sliding length increases with $\epsilon$ (Fig. 1C), our results are consistent with Eq. (1) but now there is an optimal value $\epsilon^{*}$ which minimises $\tau_{s}$. Unlike in the theory we also observe a dependence of $D_{1}$ on $\epsilon$ (Fig. 1C), which comes from the presence of energy barriers felt by the protein while sliding - in our case these are mainly due to the granularity of our polymer description, but they are likely to be present for real DNA as well, due to the modulations in the major and minor grooves, and the curvature of the DNA. Experimentally $D_{1}$ has been shown to vary over a large range of values for different conditions, and DNA sequences [4].

Intriguingly, freezing the DNA leads to a much slower search, especially for large $\epsilon$. Our simulations also show that increasing the amount of genome available in the search volume, i.e. increasing $L$ at a fixed $V$, hinders, rather than helps, facilitated diffusion, unless the affinity is very small (Fig. 1D). While the latter effect can be readily predicted from Eq. (11), understanding the difference between the frozen and moving DNA cases requires a more detailed analysis of the protein trajectories in our numerical experiment. As one might expect, the 3D search time, $\tau_{3 \mathrm{D}}$, which is dominant for small $\epsilon$, is larger ( $\mathrm{a} \sim 40 \%$ difference) for the frozen DNA; however we also observe an almost 2-fold larger value of $\tau_{1 \mathrm{D}}$ for the frozen case. Fig. $1 \mathrm{C}$ shows that while $l_{s}$ is similar for the cases of mobile and frozen DNA, $D_{1}$ changes significantly, i.e. it is smaller in the case of frozen DNA. We ascribe this difference to the fact that, when mobile, the DNA is able to adjust locally to the presence of the protein, and hence can smooth out some of the energy barriers which slow down the 1D sliding. Once the measured values of $l_{s}, D_{1}$, $A$ and $B$ [26] are put into Eq. (11), this actually provides a good fit to our data, for both mobile and frozen DNA, as shown in Fig. 1B. The small residual error may arise from the presence of the previously mentioned "intersegmental transfers", which are neglected by the theory indeed their presence somewhat changes the meaning of $l_{s}$. While traditionally $l_{s}$ is the length over which the protein "slides" during each encounter with the DNA, we here define it simply as the number of distinct DNA bead visited during the encounter - whether consecutive along the contour, or separated due to intersegmental transfers. Such events are present in our simulations, and are more common in the mobile DNA case.

The DNA conformations found in vivo in bacteria, while not yet well characterised, are likely to be quite far from those of the self-avoiding polymer normally considered in the theories for this process, and which we studied in Fig. 1. Within the prokaryotic cytosol, DNA is known to be highly looped, due to the presence of DNA-binding architectural proteins such as condensins - this helps to achieve the compaction which is required to fit the whole genome within the narrow volume of a single cell. Therefore we consider in Fig. 2 the dynamics of a protein searching for its target on a DNA which is 

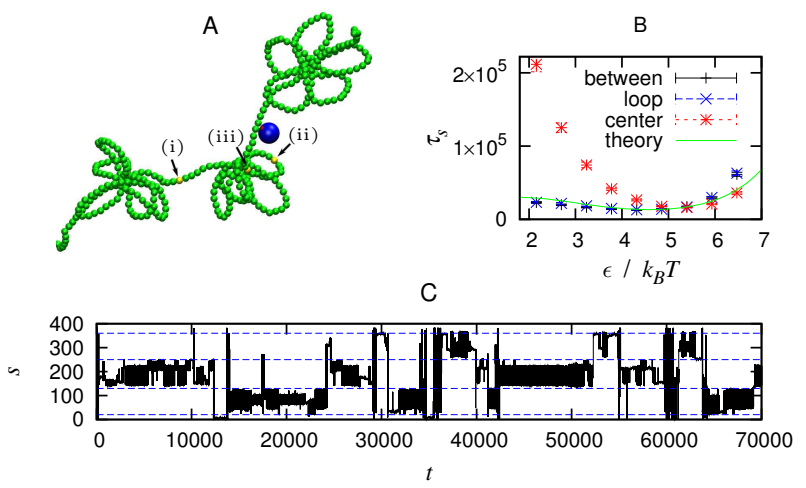

FIG. 2: (A) Snapshot of a string of rosettes. (B) Mean search time for a protein on a string of 3 rosettes with 5 loops (each of length $20 \sigma$ ) on a DNA of length $L=380 \sigma$ with $V \simeq$ $36000 \sigma^{3}$ (1\% DNA volume fraction), for different positions of the target: (i) between rosettes (bead 130), (ii) in the middle of a loop in a rosette (bead 190), and (iii) in the centre of a rosette (bead 180), as indicated in (A). The curve is the fit to the theory in Eq. 1 26]. (Choosing a different number and size of loops leads to qualitatively similar results.) (C) Time series of the DNA-bead $(s)$ nearest to the protein at a given instant, showing trapping close to a rosette centre $(\epsilon=$ $\left.5.9 k_{B} T\right)$. Dashed lines separate beads belonging to different rosettes.

made up of a string of rosettes, each of which consists of a series of loops joined together (see Fig. 2A). This idealised conformation gives a realistic local view of bacterial DNA according to a number of biological models (see e.g. [21]) and is simple enough to be included in our modelling.

Fig. 2B shows the mean search time $\tau_{s}$ as a function of $\epsilon$, for three different target positions: (i) in the centre of a rosette, (ii) in the middle of a loop in a rosette, and (iii) between rosettes. Our results show that when the affinity between the protein and the DNA is small, so that 3D diffusion dominates over 1D diffusion during the search, it takes much longer to find a target in the centre of a rosette. Such a target is more difficult to reach as the surrounding loops are in the way. Interestingly, this trend reverses for larger values of the affinity. To understand this, we observe that in the large $\epsilon$ regime each of the rosettes acts as a trap for the protein, i.e. it spends a large amount of time in a rosette, before moving to another one (see Fig. 2C). Since sliding is the dominant transport mechanism, rather than acting as a shield, the loops allow the protein to slide into the centre of the rosette. Once there intersegmental transfers are more likely to keep the protein near that centre than take it elsewhere. Such a mechanism then renders it easier to find the target if it is close to one of the traps.

Fig. 2 therefore demonstrates that DNA topology and target positioning, together with DNA-protein affinity, can be used to control the relative ease with which dif-
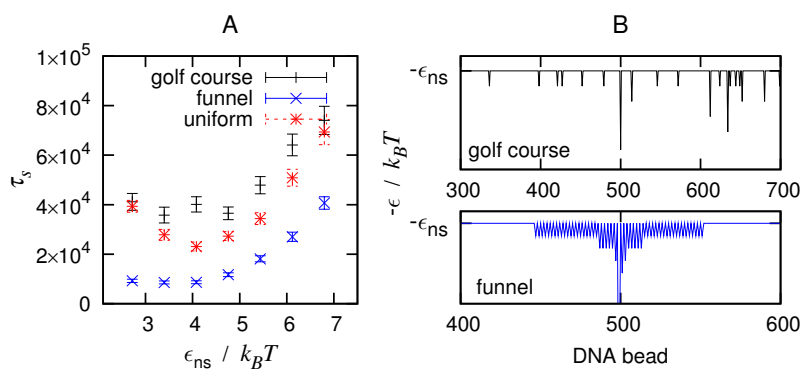

FIG. 3: (A) Plot of $\tau_{s}$ as a function of $\epsilon_{\mathrm{ns}}$ for three DNA sequences: (i) homogeneous DNA, (ii) randomly positioned traps and (iii) traps clustered so as to provide a funnel towards the target. (B) Schematic showing the DNA-protein affinity for each bead in a typical section of DNA, for the two different trap arrangements. In the funnel case traps alternate with non-specific beads. In each case the target is at bead 500, $L=1000$ and $V \simeq 10^{5} \sigma^{3}$ (1\% DNA volume fraction).

ferent regions of the genome can be accessed by proteins. We highlight that this conclusion is outside the scope of most facilitated diffusion theories based on arguments such as that in Eq. (1), in which the position of the target does not feature. More quantitatively, we have computed $\tau_{3 \mathrm{D}}$ and $\tau_{1 \mathrm{D}}$, as well as $D_{1}$, and $l_{s}$ from our data, and found that while $\tau_{3 \mathrm{D}} \sim V / D_{3} L$ still holds, it is not possible to fit $\tau_{1 \mathrm{D}}$ to the functional form $B l_{s}^{2} / D_{1}$ throughout the $\epsilon$ range considered here (not shown). This is because the rosette structure introduces large correlations between the points where the protein leaves and rejoins the DNA for each 3D excursion, meaning that $N_{s}$ is very sensitive to the target position and poorly predicted by Eq. (1) (see Fig. 2B).

We now turn to the discussion of another aspect found in real DNA and commonly neglected in theoretical work: sequence heterogeneity. The DNA sequence leads to a non-uniform free energy landscape for a protein sliding along it. In order to describe such a landscape, we allow the DNA-protein interaction to vary from one DNA bead to another, with the bead-dependent affinity set as prescribed by the model proposed in Ref. [27]. There it was postulated that there exists two possible states for a protein attached to the genome: it can either bind in a non-specific mode - with constant affinity $\epsilon_{\mathrm{ns}}$, or in a sequence-dependent, specific, mode - with affinity larger than $\epsilon_{\mathrm{ns}}$. The model in [27] assumes that the two states are in equilibrium, so the protein will be found in whichever state offers a stronger interaction. In our simulations, for DNA bead $s$ we choose a specific interaction strength $\epsilon_{\mathrm{S}}(s)$ according to an appropriate distribution [10, 27]; the affinity for that bead is then taken to be whichever is the larger of $\epsilon_{\mathrm{s}}(s)$ and $\epsilon_{\mathrm{ns}}$. In practice this leads to a free energy profile with most beads favouring the nonspecific interaction strength $\epsilon_{\mathrm{ns}}$, with a small number of "traps" with a greater interaction energy. Un- 
like those of the rosettes considered in Fig. 2, which are determined by the 3D structure of the DNA, such traps are encoded in the 1D sequence of bases.

Fig. 3 shows the dependence of $\tau_{s}$ on $\epsilon_{\mathrm{ns}}$ for a DNA chain with $L=1000 \sigma$ (corresponding to $\sim 7350$ base pairs), where the trap strength and number of traps have been determined on the basis of the statistics for the binding of a "typical" bacterial transcription factor (TF) [10, 22, 28]. We focused on the case in which the target:protein interaction energy is larger than the affinity with any of the traps, which is the most common for real TFs [10]. We compared the case of homogeneous DNA with a nonspecific interaction $\epsilon_{\mathrm{ns}}$, with two inhomogeneous sequences: (i) that in which the position of the traps is random, leading to a "golf-course" free energy landscape; and (ii) that in which the DNA sites with enhanced affinity for the proteins are clustered around the target (alternating non-specific and enhanced binding beads) so as to provide a potential funnel driving the protein to it (see Fig. 3B). We refer to these two situations as the golf-course and funnel case respectively.

A general Kramers' argument suggests that the time the protein spends in a trap may be estimated as $\tau_{\text {trap }}=$ $\tau_{0} e^{\left(\epsilon_{\text {trap }}-\epsilon_{\mathrm{ns}}\right) / k_{B} T}$, where $\tau_{0} \sim \sigma^{2} / D_{1}$ is the time it takes a protein to move from one non-specifically interacting DNA bead to the next. It is therefore not surprising that this case leads to a far larger mean search time with respect to the homogeneous DNA case, where the binding of the protein to the genome is always "non-specific" (see Fig. 3A). If the search involved 1D sliding along the DNA contour alone, one might expect that if the nonspecific interaction $\epsilon_{\mathrm{ns}}$ were increased at fixed $\epsilon_{\text {trap }}$ then this would lead to an exponential decrease in the search time (in line with the decrease in trap depth); however, for facilitated diffusion, this is balanced by the increase in $l_{s}$ (above its optimum value) which leads to a slower search.

The Kramers' argument does not apply to the funnel case, which eliminates traps other than near the target - intersegmental transfers from one "trap" to the next provide an alternative transport mechanism which avoids slowdown due to the rugged 1D potential. One may then expect that $\tau_{s}$ should be similar to the one observed with uniform DNA, with some enhancement due to the binding gradient which drives the protein towards the target once it is in its close proximity. Strikingly, the speed up with respect to the uniform case may instead reach about one order of magnitude (and more than two with respect to the golf-course case). This is probably because the presence of the funnel can decrease the likelihood of the protein being transported away from the vicinity of the target, even for small $\epsilon_{\mathrm{ns}}$ [29].

The dramatic difference between search efficiency in the golf-course and funnel case is a consequence of the assumption (from [27]) that proteins can bind to DNA either non-specifically or specifically, and the two states are in thermodynamic equilibrium so that the optimal binding for each site can be selected quickly. It is currently not clear whether this is a correct assumption - an alternative suggestion [22, 30] is that what matters may be the energy barrier between the specific and nonspecific bound states, rather than their absolute binding energy. If the energy barrier between the states was very large for all sites except the target, then our funnel sequence should not lead to much enhancement in the efficiency with respect to the random case. That is to say, the protein would see only a flat (non-specific) landscape irrespective of the sequence, and the "funnel" would not be accessible to it. It would therefore be interesting to perform in vitro single molecule experiments analogous to those of Ref. 2], where the DNA sequence is either random or designed so as to create the funnel we considered in Fig. 3. In this way one may directly test whether the predictions from our simulations hold, and hence determine which of the two theories mentioned above for DNA-protein binding applies in reality.

In conclusion, we have presented Brownian dynamics simulations of the facilitated diffusion of a protein on DNA. Unlike previous numerical work, we have focused on the impact of 3D DNA conformation and sequence heterogeneity on the search dynamics. We have found that the presence of loops in the DNA may provide a way to tune the accessibility of a target on the genome, which cannot be accounted for by existing analytical theories. By considering a string of rosettes for the DNA conformations, we have seen that when the target is in the centre of a rosette and the DNA-protein affinity is small, the time needed to find it is larger than in the case when the target is positioned between rosettes. This effect reverses for high affinity - in this regime each of the rosettes acts as a configurational trap, in the vicinity of which the protein lingers for a long time. While the conformation of prokaryotic genomes may adopt far more complicated topologies than the string of rosettes which we have considered, our results are generic in predicting a dependence on the relative positioning of loops and targets. Hence we expect they should also apply to more disordered loop networks. Finally, we have considered the case of a heterogeneous DNA, where the affinity between genome and protein is site-dependent, thereby introducing traps in the facilitated diffusion of the protein. When the sequence is random, these traps severely slow down the search process. However, when the sequence is designed so as to provide a funnel-like landscape around the target, the search may become much faster. Experiments to test this latter prediction should lead to a better understanding of the way proteins bind to DNA.

We acknowledge EPSRC grant EP/I034661/1 for funding. MEC is funded by the Royal Society. 
[1] A. D. Riggs, S. Bourgeois, M. Cohn, J. Mol. Biol. 53, 401 (1970).

[2] X. S. Xie, P. J. Choi, G. Li, N. K. Lie and G. Lia, Ann. Rev. Biophys. 37,417 (2008).

[3] J. Elf, G.-W. Li and X. S. Xie, Science 316, 1191 (2007).

[4] Y.M. Wang, R.H. Austin and E.C. Cox, Phys. Rev. Lett. 97, $048302(2006)$

[5] D. M. Gowers, G. G. Wilson, and S. E. Halford, Proc. Natl. Acad. Sci. USA 102, 15883 (2005).

[6] G.-W. Li, O. G. Berg and J. Elf, Nature Phys. 5, 294 (2009).

[7] P. H. von Hippel, O. G. Berg, J. Biol. Chem. 264, 675 (1989).

[8] S. E. Halford, J. F. Marko, Nucleic Acids Res. 32, 3040 (2004).

[9] C. Loverdo, O. Bénichou, R. Voituriez, A. Biebricher, I. Bonnet, and P. Desbiolles, Phys. Rev. Lett. 102, 188101 (2009).

[10] M. Sheinman, O. Benichou, Y. Kafri and R. Voituriez, Rep. Prog. Phys. 75, 026601 (2012).

[11] S. Condamin, O. Benichou, V. Tejedor, R. Voituriez and J. Klafter, Nature 450, 77 (2007); O. Benichou, C. Chevalier, B. Meyer and R. Voituriez, Phys. Rev. Lett. 106, 038102 (2011).

[12] R. van den Broek, M. A. Lomholt, S. M. J. Kalitz, R. Metzler and G. J. L. Wuite, Proc. Natl. Acad. Sci. USA 105, 15738 (2008); M. A. Lomholt, B. van den Broek, S-M. J. Kalisch, G. J. L. Wuite, and R. Metzler, Proc. Natl. Acad. Sci. USA 106, 8204 (2009)

[13] M. B. Elowitz, M. G. Surette, P. Wolf, J. B. Stock and S. Leiber, J. Bacteriol. 181, 197 (1999).

[14] This relies on the assumption that the point on the DNA where the protein starts a slide is not correlated with the last point of its previous interaction with the genome.

[15] K. V. Klenin, H. Merlitz, J. Langowski and C.X. Wu, Phys. Rev. Lett. 96, 018104 (2006); H. Merlitz, K. V. Klenin and J. Langowski, J. Chem. Phys. 124, 134908 (2006); J. Chem. Phys. 125, 041906 (2006).

[16] M. Sheinman and Y. Kafri, Phys. Biol. 6, 0160033 (2009).

[17] A.-M. Florescu and M. Joyeux, J. Chem. Phys. 130, 015103 (2009).

[18] T. Schotz, R. A. Neher and U. Gerland, Phys. Rev. E 84, 051911 (2011).

[19] G. Foffano, D. Marenduzzo and E. Orlandini, Phys. Rev. E 85, 021919 (2012).

[20] E. P. C. Rocha, Annu. Rev. Genet. 42, 211 (2008).

[21] P. R. Cook, Principles of Nuclear Structure and Function, Wiley-Liss (New York) (2001).

[22] M. Slutsky and L. A. Mirny, Biophys. J. 87, 4021 (2004).

[23] O. Kratky, G. Porod, Rec. Trav. Chim. Pays-Bas. 68, 1106 (1949).

[24] In our simulations we fixed the following parameters: $m_{i}=1$ for a DNA-bead, and $m_{i}=3$ for a protein (core plus patch); likewise $\gamma_{i}=1$ for a DNA-bead and $\gamma_{i}=3$ for a protein. The latter choice defines the viscosity $\eta$ of the fictitious Brownian fluid, and the diameter $d$ of the particles, via the usual Stokes-Einstein relation $\gamma=3 \pi \eta d$. We use a cubic simulation box with periodic boundary conditions (this avoids complications due to interactions between the DNA or proteins and the boundaries). Systems are equilibrated for at least $10^{6}$ time steps before the attractive interactions are switched on, so the initial DNA configuration and protein position are random. To map timescales to physical units, we note a time unit in Figs. 1-3 corresponds to $36 \mathrm{~ns}$ (if $\eta=1 \mathrm{cP}$ ).

[25] S. J. Plimpton, J. Comp. Phys. 117, 1 (1995) (http://lammps.sandia.gov).

[26] To fit our data with the theory in Eq. (1), we need to estimate the constants $A$ and $B$, by finding $\tau_{3 \mathrm{D}}$ and $\tau_{1 \mathrm{D}}$ from the numerical trajectories. From the known values of $V, L$ and $D_{3}$ we fit the $\tau_{3 \mathrm{D}}$ data to find $A$. From a Kramers' argument we expect that $\tau_{1 \mathrm{D}}$ will depend exponentially on the DNA-protein affinity $\epsilon$; fitting gives a function $\tau_{1 \mathrm{D}}(\epsilon)$. To find $N_{s}$ and the constant $B$ we need to know $D_{1}$ and $l_{s}$, both of which are found to depend on $\epsilon$. To estimate the $1 \mathrm{D}$ diffusion we use the statistics of the sliding events; i.e. we calculate the diffusion constant for each value of $\epsilon$ from the mean square 1D displacement (along the DNA) versus time, and then fit to find a function $D_{1}(\epsilon)$. Finally, $B$ is estimated by using the previously found expressions for $\tau_{1 \mathrm{D}}(\epsilon)$ and $D_{1}(\epsilon)$, and fitting $l_{s}$ (measured from the trajectories) to the equation $l_{s}=B^{-1 / 2} \sqrt{\tau_{1 \mathrm{D}}(\epsilon) D_{1}(\epsilon)}$.

[27] U Gerland, J. D. Moroz and T. Hwa, Proc. Natl. Acad. Sci. USA 99, 12015 (2002).

[28] A given protein can be characterised by the width, $w$, of the distribution of binding affinities for different DNA sites [10]. Transcription factors typically have $w$ in the range $3-8 k_{B} T$; we present results for $w=3 k_{B} T$. This typically leads to 50 traps on a DNA of length 1000 beads, so the average trap separation is 20 beads. It should be noted that the average sliding length is $<20 \sigma$ even for the largest values of $\epsilon$ examined. Therefore during the majority of slides the TF does not encounter a trap. Similar results are found for e.g. $w=4.1 k_{B} T$, although the slowdown in the golf-course case is about an order of magnitude larger (and does not depend much on $\epsilon_{\mathrm{ns}}$ ).

[29] This interpretation is corroborated by further simulations in which we shuffled the position of the traps within the funnel region. Fewer search rounds are required to find the target than in the uniform case, and the search time is always less than the golf course case. However, the gain is less than the unshuffled funnel as this configuration provides no binding energy gradient.

[30] O. Benichou, Y. Kafri, M. Sheinman and R. Voituriez, Phys. Rev. Lett. 103, 138102 (2009). 